\title{
Some Notes about Efficiency Balanced Block Designs with Repeated Blocks
}

\author{
Bronisław Ceranka and Małgorzata Graczyk ${ }^{1}$
}

\begin{abstract}
Various problems for a class of efficiency balanced block designs based on balanced incomplete block designs with repeated blocks are considered.
\end{abstract}

\section{Introduction}

Let us consider a class of block designs in which $v$ treatments are arranged in $b$ blocks according to the incidence matrix $\mathbf{N}=\left(n_{i j}\right), n_{i j}$ denotes the number of experimental units in the $j$ th block getting the $i$ th treatment, $i=1,2, \ldots, v, j=1,2, \ldots, b$. The $i$ th treatment is replicated $r_{i}$ times and the size of $j$ th block is $k_{j}, \mathbf{r}=\left[\begin{array}{llll}r_{1} & r_{2} & \ldots & r_{v}\end{array}\right]^{\prime}, \mathbf{k}=\left[\begin{array}{llll}k_{1} & k_{2} & \ldots & k_{b}\end{array}\right]^{\prime}$. If $r_{1}=r_{2}=\ldots=r_{v}$, then the design is called equireplicated. In the course of performing an experiment using a block design, a cost is often attached to each block. Thus it is of interest having the property that each pair of distinct treatments appear in the same, or nearly the same, number of blocks. The set of all distinct blocks in a block design is called support and denoted as $b^{*}$. The constructions of the designs admitting block designs with repeated blocks are available in the literature (Ceranka and Graczyk, 2007).

Balancing is a desirable property of any block design. There are balanced designs of various types.

Definition 1 A block design is called variance balanced (VB) if every normalized estimable linear function of treatment effects is estimated with the same variance.

Let us consider the matrix

$$
\mathbf{C}=\mathbf{R}-\mathbf{N} \mathbf{K}^{-1} \mathbf{N}^{\prime}
$$

where $\mathbf{R}=\operatorname{diag}\left\{r_{1} r_{2} \ldots r_{v}\right\}, \mathbf{K}=\operatorname{diag}\left\{k_{1} k_{2} \ldots k_{b}\right\}$.

Kageyama (1974) showed that $\mathbf{N}$ is a VB block design if and only if

$$
\mathbf{C}=\rho\left(\mathbf{I}_{v}-\frac{1}{v} \mathbf{1}_{v} \mathbf{1}_{v}^{\prime}\right)
$$

\footnotetext{
${ }^{1}$ Department of Mathematical and Statistical Methods, Poznan University of Life Sciences, Wojska Polskiego 28, 60-637 Poznań, Poland; bronicer@au.poznan.pl, magra@au.poznan.pl
} 
where $\rho$ is the unique nonzero eigenvalue of $\mathbf{C}$ with multiplicity $v-1, \mathbf{I}_{v}$ is the unit matrix of order $v, \mathbf{1}_{v}$ is $v \times 1$ vector all of whose elements are 1 .

Let us consider the matrix $\mathbf{M}_{0}$ given by Caliński (1971)

$$
\mathbf{M}_{0}=\mathbf{R}^{-1} \mathbf{N K}^{-1} \mathbf{N}^{\prime}-\frac{1}{n} \mathbf{1}_{v} \mathbf{r}^{\prime}
$$

where $n$ is total number of experimental units and $n=\sum_{i=1}^{v} r_{i}=\sum_{j=1}^{b} k_{j}$.

Definition 2 A block design is called efficiency balanced (EB) if all treatments contrasts $\mathbf{s}^{\prime} \mathbf{T}\left(\mathbf{r}^{\prime} \mathbf{s}=0\right)$ satisfy

$$
\mathbf{M}_{0} \mathbf{s}=\mu \mathbf{s}
$$

where $\mathbf{T}=\left[T_{1} T_{2} \ldots T_{v}\right]^{\prime}, T_{i}$ is the total yield for the $i$ th treatment, $\mu$ is the unique nonzero eigenvalue of $\mathbf{M}_{0}$ with multiplicity $v-1$ and $\mathbf{M}_{0}$ is given in (1.3).

Caliński (1971) showed that for such designs, every treatment contrast is estimated with the same efficiency $1-\mu$ and $\mathbf{N}$ is a EB block design if and only if

$$
\mathbf{M}_{0}=\mu\left(\mathbf{I}_{v}-\frac{1}{n} \mathbf{1}_{v} \mathbf{r}^{\prime}\right) .
$$

Kageyama (1980) proved that for the EB block design $\mathbf{N}$, equation (1.4) is fulfilled if and only if

$$
\mathbf{C}=(1-\mu)\left(\mathbf{R}-\frac{1}{n} \mathbf{r r}^{\prime}\right)
$$

In the paper we present new construction methods of EB block designs with repeated blocks for $v$ treatments and some ways of admitting given design structures to construct new designs for other numbers of treatments.

\section{Construction for $v_{1}<v$ treatments}

In order to construct a EB block design, we consider the VB block design, $\mathbf{N}$ with repeated blocks with parameters $v, b, r, \mathbf{k}, b^{*}$ and the $v_{1} \times v$ matrix $\mathbf{E}=\left(e_{l t}\right)$, where for each $t, e_{l t}=1$ for a certain $l, e_{l^{\prime} t}=0, l \neq l^{\prime}, l, l^{\prime}=1,2, \ldots, v_{1}, t=1,2, \ldots, v$, i.e., $\mathbf{E}^{\prime} \mathbf{1}_{v_{1}}=\mathbf{1}_{v}$.

Now, we form the matrix

$$
\mathbf{N}_{1}=\mathbf{E N}
$$

From the design matrix $\mathbf{N}$ for $v$ treatments, we construct the design matrix $\mathbf{N}_{1}$ for $v_{1}$ treatments, $v_{1}<v$. Hence we have

$$
\mathbf{N}_{1} \mathbf{1}_{b}=\mathbf{E N} \mathbf{1}_{b}=r \mathbf{E} \mathbf{1}_{b}=\mathbf{r}_{1}, \mathbf{N}_{1}^{\prime} \mathbf{1}_{v_{1}}=\mathbf{N}^{\prime} \mathbf{E}^{\prime} \mathbf{1}_{v_{1}}=\mathbf{N}^{\prime} \mathbf{1}_{v}=\mathbf{k}=\mathbf{k}_{1}, n=n_{1} .
$$


Theorem 1 If $\mathbf{N}$ is the equireplicated VB block design with repeated blocks, then $\mathbf{N}_{1}$ given in the form (2.1) is a EB block design with repeated blocks and with the parameters $v_{1}, b_{1}=b, \mathbf{r}_{1}, \mathbf{k}_{1}=\mathbf{k}, b_{1}^{*}=b^{*}$.

Proof. In any equireplicated design $n=r v$. From (1.1) and (1.2), for the VB block design $\mathrm{N}$, we have

$$
\begin{aligned}
\mathbf{C}_{1}= & \mathbf{E} \mathbf{C E}^{\prime}=\mathbf{E}\left(\rho\left(\mathbf{I}_{v}-\frac{1}{v} \mathbf{1}_{v} \mathbf{1}_{v}^{\prime}\right)\right) \mathbf{E}^{\prime} \\
& =\frac{\rho}{r} \mathbf{E}\left(r \mathbf{I}_{v}-\frac{r^{2}}{n} \mathbf{1}_{v} \mathbf{1}_{v}^{\prime}\right) \mathbf{E}^{\prime} \\
& =\frac{\rho}{r} \mathbf{E}\left(r \mathbf{I}_{v}-\frac{1}{n} \mathbf{r} \mathbf{r}^{\prime}\right) \mathbf{E}^{\prime} \\
& =\frac{\rho}{r}\left(\mathbf{R}_{1}-\frac{1}{n_{1}} \mathbf{r}_{1} \mathbf{r}_{1}^{\prime}\right)
\end{aligned}
$$

where $\mu=\frac{r-\rho}{r}$.

Hence, the Theorem is proven.

Theorem 2 If $\mathbf{N}$ is the EB block design with repeated blocks, then $\mathbf{N}_{1}$ given in the form (2.1) is a EB block design with repeated blocks.

Proof. From (1.5) for the EB block design N, we have

$$
\mathbf{C}_{1}=\mathbf{E} \mathbf{C E} \mathbf{E}^{\prime}=(1-\mu) \mathbf{E}\left(\mathbf{R}-\frac{1}{n} \mathbf{r r}^{\prime}\right) \mathbf{E}^{\prime}=(1-\mu)\left(\mathbf{R}_{1}-\frac{1}{n_{1}} \mathbf{r}_{1} \mathbf{r}_{1}^{\prime}\right)
$$

Hence the Theorem is proven.

For example, let us consider the VB block design with repeated blocks and parameters $v=9, b=25, r=9, \mathbf{k}=\left[\begin{array}{ll}3 \cdot \mathbf{1}_{24} & 9\end{array}\right]^{\prime}, b^{*}=13$ (Example 2.7. Ceranka and Graczyk, 2007),

$$
\mathbf{N}=\left[\begin{array}{lllllllllllllllllllllllll}
1 & 1 & 1 & 1 & 0 & 0 & 0 & 0 & 0 & 0 & 0 & 0 & 1 & 1 & 1 & 1 & 0 & 0 & 0 & 0 & 0 & 0 & 0 & 0 & 1 \\
1 & 0 & 0 & 0 & 1 & 1 & 1 & 0 & 0 & 0 & 0 & 0 & 1 & 0 & 0 & 0 & 1 & 1 & 1 & 0 & 0 & 0 & 0 & 0 & 1 \\
0 & 1 & 0 & 0 & 1 & 0 & 0 & 1 & 1 & 0 & 0 & 0 & 0 & 1 & 0 & 0 & 1 & 0 & 0 & 1 & 1 & 0 & 0 & 0 & 1 \\
0 & 0 & 1 & 0 & 0 & 1 & 0 & 1 & 0 & 1 & 0 & 0 & 0 & 0 & 1 & 0 & 0 & 1 & 0 & 1 & 0 & 1 & 0 & 0 & 1 \\
0 & 0 & 0 & 1 & 0 & 0 & 1 & 1 & 0 & 0 & 1 & 0 & 0 & 0 & 0 & 1 & 0 & 0 & 1 & 1 & 0 & 0 & 1 & 0 & 1 \\
1 & 0 & 0 & 0 & 0 & 0 & 0 & 0 & 1 & 1 & 1 & 0 & 1 & 0 & 0 & 0 & 0 & 0 & 0 & 0 & 1 & 1 & 1 & 0 & 1 \\
0 & 1 & 0 & 0 & 0 & 0 & 1 & 0 & 0 & 1 & 0 & 1 & 0 & 1 & 0 & 0 & 0 & 0 & 1 & 0 & 0 & 1 & 0 & 1 & 1 \\
0 & 0 & 1 & 0 & 1 & 0 & 0 & 0 & 0 & 0 & 1 & 1 & 0 & 0 & 1 & 0 & 1 & 0 & 0 & 0 & 0 & 0 & 1 & 1 & 1 \\
0 & 0 & 0 & 1 & 0 & 1 & 0 & 0 & 1 & 0 & 0 & 1 & 0 & 0 & 0 & 1 & 0 & 1 & 0 & 0 & 1 & 0 & 0 & 1 & 1
\end{array}\right] ;
$$

and we consider the matrix

$$
\mathbf{E}=\left[\begin{array}{lllllllll}
1 & 1 & 0 & 0 & 0 & 0 & 0 & 0 & 0 \\
0 & 0 & 1 & 1 & 0 & 0 & 0 & 0 & 0 \\
0 & 0 & 0 & 0 & 1 & 1 & 0 & 0 & 0 \\
0 & 0 & 0 & 0 & 0 & 0 & 1 & 0 & 0 \\
0 & 0 & 0 & 0 & 0 & 0 & 0 & 1 & 0 \\
0 & 0 & 0 & 0 & 0 & 0 & 0 & 0 & 1
\end{array}\right]
$$


Thus the matrix $\mathbf{N}_{1}$ in the form (2.1) is given as

$$
\mathbf{N}_{1}=\left[\begin{array}{lllllllllllllllllllllllll}
2 & 1 & 1 & 1 & 1 & 1 & 1 & 0 & 0 & 0 & 0 & 0 & 2 & 1 & 1 & 1 & 1 & 1 & 1 & 0 & 0 & 0 & 0 & 0 & 2 \\
0 & 1 & 1 & 0 & 1 & 1 & 0 & 2 & 1 & 1 & 0 & 0 & 0 & 1 & 1 & 0 & 1 & 1 & 0 & 2 & 1 & 1 & 0 & 0 & 2 \\
1 & 0 & 0 & 1 & 0 & 0 & 1 & 1 & 1 & 1 & 2 & 0 & 1 & 0 & 0 & 1 & 0 & 0 & 1 & 1 & 1 & 1 & 2 & 0 & 2 \\
0 & 1 & 0 & 0 & 0 & 0 & 1 & 0 & 0 & 1 & 0 & 1 & 0 & 1 & 0 & 0 & 0 & 0 & 1 & 0 & 0 & 1 & 0 & 1 & 1 \\
0 & 0 & 1 & 0 & 1 & 0 & 0 & 0 & 0 & 0 & 1 & 1 & 0 & 0 & 1 & 0 & 1 & 0 & 0 & 0 & 0 & 0 & 1 & 1 & 1 \\
0 & 0 & 0 & 1 & 0 & 1 & 0 & 0 & 1 & 0 & 0 & 1 & 0 & 0 & 0 & 1 & 0 & 1 & 0 & 0 & 1 & 0 & 0 & 1 & 1
\end{array}\right] .
$$

Here, $\mathbf{N}_{1}$ is the matrix of a EB block design with repeated blocks for $v_{1}=6$ objects. In this design, $b_{1}=b=25, \mathbf{r}_{1}=\left[\begin{array}{lll}18 \cdot \mathbf{1}_{3}^{\prime} & 9 \cdot \mathbf{1}_{3}^{\prime}\end{array}\right]^{\prime}, \mathbf{k}_{1}=\mathbf{k}=\left[\begin{array}{lll}3 \cdot \mathbf{1}_{24} & 9\end{array}\right]^{\prime}$ and $b_{1}^{*}=b^{*}=13$.

For the design $\mathbf{N}_{1}$, we have

$$
\mathbf{C}_{1}=\frac{7}{9}\left[\begin{array}{rrrrrr}
14 & -4 & -4 & -2 & -2 & -2 \\
-4 & 14 & -4 & -2 & -2 & -2 \\
-4 & -4 & 14 & -2 & -2 & -2 \\
-2 & -2 & -2 & 8 & -1 & -1 \\
-2 & -2 & -2 & -1 & 8 & -1 \\
-2 & -2 & -2 & -1 & -1 & 8
\end{array}\right]
$$

and $1-\mu=\frac{2}{9}$. Thus it is easy to see that $\mathbf{C}_{1}$ is given in the form (1.5).

On the other hand,

$$
\mathbf{M}_{0}=\frac{2}{9}\left(\frac{1}{9}\left[\begin{array}{rrrrrr}
7 & -2 & -2 & -1 & -1 & -1 \\
-2 & 7 & -2 & -1 & -1 & -1 \\
-2 & -2 & 7 & -1 & -1 & -1 \\
-2 & -2 & -2 & 8 & -1 & -1 \\
-2 & -2 & -2 & -1 & 8 & -1 \\
-2 & -2 & -2 & -1 & -1 & 8
\end{array}\right]\right)
$$

and its eigenvalues are 0 and $\mu=\frac{2}{9}$ with the multiplicity 5 . Hence, $\mathbf{N}_{1}$ is EB.

\section{$3 \quad$ Construction for $v$ treatments}

We consider the set of $\xi$ EB block designs with repeated blocks $\mathbf{N}_{s}$ with the parameters $v, b_{s}, \mathbf{r}_{s}, \mathbf{k}_{s}, b_{s}^{*}, s=1,2, \ldots, \xi$. We form the matrix

$$
\mathbf{N}=\left[\begin{array}{llll}
\mathbf{N}_{1} & \mathbf{N}_{2} & \ldots & \mathbf{N}_{\xi}
\end{array}\right]
$$

Theorem 3 If $\mathbf{N}_{s}$ is a EB block design with repeated blocks, $s=1,2, \ldots, \xi$, for which vectors of treatment replications are mutually proportional, then $\mathbf{N}$ in the form (3.1) is a EB block design with repeated blocks with the parameters $v, b=\sum_{s=1}^{\xi} b_{s}, \mathbf{r}=$ $\sum_{s=1}^{\xi} \mathbf{r}_{s}, \mathbf{k}=\left[\begin{array}{llll}\mathbf{k}_{1} & \mathbf{k}_{2} & \ldots \mathbf{k}_{\xi}\end{array}\right]^{\prime}, b^{*} \leq \sum_{s=1}^{\xi} b_{s}^{*}$. 
Proof. For EB block designs with repeated blocks $\mathbf{N}_{s}$, the vectors of treatment replications are mutually proportional and we have $\mathbf{r}_{s}=a_{s} \mathbf{r}, \sum_{s=1}^{\xi} a_{s}=1$ and $\mathbf{r}=\sum_{s=1}^{\xi} \mathbf{r}_{s}, s=$ $1,2, \ldots, \xi$. Hence, for the matrix $\mathbf{C}$ in (1.1) and taking into consideration (1.5), we have

$$
\begin{aligned}
\mathbf{C} & =\sum_{s=1}^{\xi}\left(\mathbf{R}_{s}-\mathbf{N}_{s} \mathbf{K}_{s}^{-1} \mathbf{N}_{\mathbf{s}}^{\prime}\right) \\
& =\sum_{s=1}^{\xi}\left(1-\mu_{s}\right)\left(\mathbf{R}_{s}-\frac{1}{n_{s}} \mathbf{r}_{s} \mathbf{r}_{s}^{\prime}\right) \\
& =\sum_{s=1}^{\xi}\left(1-\mu_{s}\right)\left(a_{s} \mathbf{R}-\frac{1}{a_{s} n} a_{s}^{2} \mathbf{r} \mathbf{r}^{\prime}\right) \\
& =\mathbf{R} \sum_{s=1}^{\xi} a_{s}\left(1-\mu_{s}\right)-\frac{1}{n} \sum_{s=1}^{\xi}\left(1-\mu_{s}\right) a_{s} \mathbf{r} \mathbf{r}^{\prime} \\
& =\mathbf{R}\left(\sum_{s=1}^{\xi} a_{s}-\sum_{s=1}^{\xi} a_{s} \mu_{s}\right)-\frac{1}{n} \mathbf{r r}^{\prime}\left(\sum_{s=1}^{\xi} a_{s}-\sum_{s=1}^{\xi} a_{s} \mu_{s}\right) \\
& =(1-\mu)\left(\mathbf{R}-\frac{1}{n} \mathbf{r r}^{\prime}\right) .
\end{aligned}
$$

Thus we have required result.

Corollary 1 If $\mathbf{N}_{s}$ and $\mathbf{N}_{w}$ are EB block designs with repeated blocks, $s, w=$ $1,2, \ldots, \xi, s \neq w$, for which vectors of treatment replications are mutually proportional, then

$$
a_{s}\left(1-\mu_{s}\right) \mathbf{C}_{w}=a_{w}\left(1-\mu_{w}\right) \mathbf{C}_{s}
$$

where $a_{s}$ and $a_{w}$ are some constants.

For example, let us consider the EB block designs with repeated blocks and with the parameters $v=4, b_{1}=10, \mathbf{r}_{1}=\left[\begin{array}{ll}8 & 4 \cdot \mathbf{1}_{3}^{\prime}\end{array}\right]^{\prime}, k_{1}=2, b_{1}^{*}=7 \quad$ and $v=4, b_{2}=$ $10, \mathbf{r}_{2}=\left[\begin{array}{ll}12 & 6 \cdot \mathbf{1}_{3}^{\prime}\end{array}\right]^{\prime}, k_{2}=3, b_{2}^{*}=7$ given by the incidence matrices

$$
\begin{aligned}
& \mathbf{N}_{1}=\left[\begin{array}{llllllllll}
2 & 1 & 1 & 1 & 1 & 1 & 1 & 0 & 0 & 0 \\
0 & 1 & 0 & 0 & 1 & 0 & 0 & 1 & 1 & 0 \\
0 & 0 & 1 & 0 & 0 & 1 & 0 & 1 & 0 & 1 \\
0 & 0 & 0 & 1 & 0 & 0 & 1 & 0 & 1 & 1
\end{array}\right] \text { and } \\
& \mathbf{N}_{2}=\left[\begin{array}{llllllllll}
0 & 1 & 1 & 1 & 1 & 1 & 1 & 2 & 2 & 2 \\
1 & 0 & 1 & 1 & 0 & 1 & 1 & 0 & 0 & 1 \\
1 & 1 & 0 & 1 & 1 & 0 & 1 & 0 & 1 & 0 \\
1 & 1 & 1 & 0 & 1 & 1 & 0 & 1 & 0 & 0
\end{array}\right]
\end{aligned}
$$

Based on the matrices $\mathbf{N}_{1}$ and $\mathbf{N}_{2}$, we form the design matrix $\mathbf{N}$ in the form (3.1) as

$$
\mathbf{N}=\left[\begin{array}{llllllllllllllllllll}
2 & 1 & 1 & 1 & 1 & 1 & 1 & 0 & 0 & 0 & 0 & 1 & 1 & 1 & 1 & 1 & 1 & 2 & 2 & 2 \\
0 & 1 & 0 & 0 & 1 & 0 & 0 & 1 & 1 & 0 & 1 & 0 & 1 & 1 & 0 & 1 & 1 & 0 & 0 & 1 \\
0 & 0 & 1 & 0 & 0 & 1 & 0 & 1 & 0 & 1 & 1 & 1 & 0 & 1 & 1 & 0 & 1 & 0 & 1 & 0 \\
0 & 0 & 0 & 1 & 0 & 0 & 1 & 0 & 1 & 1 & 1 & 1 & 1 & 0 & 1 & 1 & 0 & 1 & 0 & 0
\end{array}\right]
$$


Here, $\mathbf{N}$ is the incidence matrix of the EB block design with the parameters $v=$ $4, b=20, \mathbf{r}=\left[\begin{array}{ll}20 & 10 \cdot \mathbf{1}_{3}^{\prime}\end{array}\right]^{\prime}, \mathbf{k}=\left[\begin{array}{lll}2 \cdot \mathbf{1}_{10}^{\prime} & 3 \cdot \mathbf{1}_{10}^{\prime}\end{array}\right]^{\prime}, b^{*}=14$. For this design, we have

$$
\mathbf{C}=\frac{3}{4}\left(\frac{8}{3}\left[\begin{array}{rrrr}
6 & -2 & -2 & -2 \\
-2 & 4 & -1 & -1 \\
-2 & -1 & 4 & -1 \\
-2 & -1 & -1 & 4
\end{array}\right]\right)
$$

and $1-\mu=\frac{3}{4}$. Thus it is easy to see that $\mathbf{C}$ is given in (1.5). On the other hand,

$$
\mathbf{M}_{0}=\frac{1}{4}\left(\frac{1}{5}\left[\begin{array}{rrrr}
3 & -1 & -1 & -1 \\
-2 & 4 & -1 & -1 \\
-2 & -1 & 4 & -1 \\
-2 & -1 & -1 & 4
\end{array}\right]\right)
$$

and its eigenvalues are 0 and $\mu=\frac{1}{4}$ with the multiplicity 3 . Hence, $\mathbf{N}$ is EB.

\section{Construction for $v+1$ treatments}

Let $\mathbf{N}_{\eta}, \eta=1,2$, be the incidence matrix of the balanced incomplete block design with repeated blocks with the parameters $v, b_{\eta}, r_{\eta}, k_{\eta}, \lambda_{\eta}, b_{\eta}^{*}$. Now, we form the matrix $\mathbf{N}$ as

$$
\mathbf{N}=\left[\begin{array}{cc}
\mathbf{N}_{1} \otimes \mathbf{1}_{t}^{\prime} & \mathbf{N}_{2} \otimes \mathbf{1}_{u}^{\prime} \\
d \mathbf{1}_{b_{1}}^{\prime} \otimes \mathbf{1}_{t}^{\prime} & \mathbf{0}_{b_{2}}^{\prime} \otimes \mathbf{1}_{u}^{\prime}
\end{array}\right]
$$

Theorem 4 A block design with an incidence matrix $\mathbf{N}$ of the form (4.1) is a EB block design with repeated blocks with the parameters $v+1, b=t b_{1}+u b_{2}$,

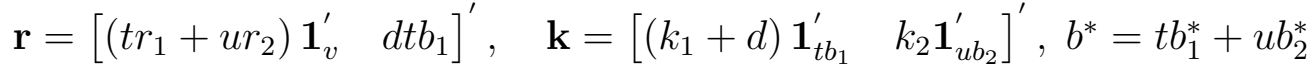
if and only if the constants $t, u$ and $d$ satisfy the equalities

$$
t\left(r_{1}-\lambda_{1}\right) k_{2}=u\left(v d \lambda_{2}-k_{1}\left(r_{2}-\lambda_{2}\right)\right)
$$

and

$$
d>\frac{k_{1}\left(r_{2}-\lambda_{2}\right)}{v \lambda_{2}}
$$

Proof. For the block design with the matrix $\mathbf{N}$ given in (4.1), we have

$$
\mathbf{C}=\left[\begin{array}{cc}
{\left[t\left(r_{1}-\frac{r_{1}-\lambda_{1}}{k_{1}+d}\right)+u\left(r_{2}-\frac{r_{2}-\lambda_{2}}{k_{2}}\right)\right] \mathbf{I}_{v}-\left[\frac{t \lambda_{1}}{k_{1}+d}+\frac{u \lambda_{2}}{k_{2}}\right] \mathbf{1}_{v} \mathbf{1}_{v}^{\prime}} & -\frac{d t r_{1}}{k_{1}+d} \mathbf{1}_{v} \\
-\frac{d t r_{1}}{k_{1}+d} \mathbf{1}_{v}^{\prime} & \frac{d t b_{1} k_{1}}{k_{1}+d}
\end{array}\right]
$$


Now, we compare the matrix $\mathbf{C}$ of the form (1.5) and (2.6). We have

$$
\frac{t \lambda_{1}}{k_{1}+d}+\frac{u \lambda_{2}}{k_{2}}=\frac{\left(t r_{1}+u r_{2}\right)^{2}}{n}
$$

and

$$
\frac{d t r_{1}}{k_{1}+d}=\frac{\left(t r_{1}+u r_{2}\right) d t b_{1}}{n} .
$$

If the conditions (4.2) and (4.3) hold, then the matrix $\mathbf{C}$ is of the form (1.5), where $1-\mu=\frac{k_{1}\left(t r_{1}+u r_{2}\right)+d t r_{1}}{\left(k_{1}+d\right)\left(t r_{1}+u r_{2}\right)}$. So, the Theorem is proven.

In particular case when $t=u=1$, we have

Corollary 2. A block design with the incidence matrix $\mathbf{N}$ of the form

$$
\mathbf{N}=\left[\begin{array}{cc}
\mathbf{N}_{1} & \mathbf{N}_{2} \\
d \mathbf{1}_{b_{1}}^{\prime} & \mathbf{0}_{b_{2}}^{\prime}
\end{array}\right]
$$

is a EB block design with repeated blocks with the parameters $v+1, \quad b=b_{1}+b_{2}$, $\mathbf{r}=\left[\begin{array}{ll}\left(r_{1}+r_{2}\right) \mathbf{1}_{v}^{\prime} & d b_{1}\end{array}\right]^{\prime}, \quad \mathbf{k}=\left[\begin{array}{ll}\left(k_{1}+d\right) \mathbf{1}_{b_{1}}^{\prime} & k_{2} \mathbf{1}_{b_{2}}^{\prime}\end{array}\right]^{\prime}, b^{*}=b_{1}^{*}+b_{2}^{*} \quad$ if and only if

$$
\left(r_{1}-\lambda_{1}\right) k_{2}=v \lambda_{2} d-k_{1}\left(r_{2}-\lambda_{2}\right) \quad \text { and } \quad d>\frac{k_{1}\left(r_{2}-\lambda_{2}\right)}{v \lambda_{2}} .
$$

As an example, let us consider the balanced incomplete block design with the parameters $v=7, \quad b_{1}=28, \quad r_{1}=12, \quad k_{1}=3, \quad \lambda_{1}=4, \quad b_{1}^{*}=11$ given by the incidence matrix

$$
\mathbf{N}_{1}=\left[\begin{array}{c}
111111000000111100000000110 \\
111000111000000011110000101 \\
111000000111000000001111011 \\
000000111111111100000000011 \\
0001110001110000111100000101 \\
0001111110000000000011111001 \\
000000000000111111111110000
\end{array}\right]
$$

and

$v=7, b_{2}=21, r_{2}=9, k_{2}=3, \lambda_{2}=3, b_{2}^{*}=7$ given by the incidence matrix

$$
\mathbf{N}_{2}=\left[\begin{array}{c}
111000011100001110000 \\
100110010011001001100 \\
010101001010100101010 \\
100001110000111000011 \\
001100100110010011001 \\
001011000101100010110 \\
010010101001010100101
\end{array}\right]
$$

Hence $d=2$, and $\mathbf{N}$ in (4.1) given as 


$$
\mathbf{N}=\left[\begin{array}{c}
11111000000111100000000110111000011100001110000 \\
111000111000000011110000101100110010011001001100 \\
111000000111000000001111011010101001010100101010 \\
000000111111111100000000011100001110000111000011 \\
0001110001110000111100000101001100100110010011001 \\
0001111110000000000011111001001011000101100010110 \\
0000000000001111111111110000010010101001010100101
\end{array}\right]
$$

is the incidence matrix of the EB block design with the parameters $v=7, b=49, r=$ $21, k=3, b^{*}=18$. For the design $\mathbf{N}$, we have

$$
\mathbf{C}=\frac{7}{9}\left(3\left[\begin{array}{rrrrrrr}
6 & -1 & -1 & -1 & -1 & -1 & -1 \\
-1 & 6 & -1 & -1 & -1 & -1 & -1 \\
-1 & -1 & 6 & -1 & -1 & -1 & -1 \\
-1 & -1 & -1 & 6 & -1 & -1 & -1 \\
-1 & -1 & -1 & -1 & 6 & -1 & -1 \\
-1 & -1 & -1 & -1 & -1 & 6 & -1 \\
-1 & -1 & -1 & -1 & -1 & -1 & 6
\end{array}\right]\right)
$$

and $\quad 1-\mu=\frac{7}{9}$.

Thus it is easy to see that $\mathbf{C}$ is given in the form (1.5). On the other hand,

$$
\mathbf{M}_{0}=\frac{2}{9}\left(\frac{1}{7}\left[\begin{array}{rrrrrrr}
6 & -1 & -1 & -1 & -1 & -1 & -1 \\
-1 & 6 & -1 & -1 & -1 & -1 & -1 \\
-1 & -1 & 6 & -1 & -1 & -1 & -1 \\
-1 & -1 & -1 & 6 & -1 & -1 & -1 \\
-1 & -1 & -1 & -1 & 6 & -1 & -1 \\
-1 & -1 & -1 & -1 & -1 & 6 & -1 \\
-1 & -1 & -1 & -1 & -1 & -1 & 6
\end{array}\right]\right)
$$

its eigenvalues are 0 , and $\mu=\frac{2}{9}$ with the multiplicity 6 . Hence, $\mathbf{N}$ is EB.

\section{References}

[1] Caliński, T. (1971): On some desirable patterns in block designs. Biometrics, 27, 275-292.

[2] Ceranka, B. and Graczyk, M. (2007): Variance balanced block designs with repeated blocks. Applied Mathematical Sciences, Hikari Ltd., 1, 2727-2734.

[3] Kageyama, S. (1974): Reduction of associate classes for block designs and releated combinatorial arrangements. Hiroshima Math. J. , 4, 527-618.

[4] Kageyama, S. (1974): On properties of efficiency - balanced designs. Commun. Statist. - Theor. Meth. , A 9, 597-616. 